\title{
BMJ Open High haemoglobin levels and mortality in males with intracerebral haemorrhage: a retrospective cohort study
}

\author{
Shuting Zhang (D) , ${ }^{1}$ Yang Shu, ${ }^{2}$ Wenjing Li, ${ }^{3}$ Chenchen Wei (D) , ${ }^{1}$ Aiping Deng, ${ }^{4}$ \\ Yajun Cheng, ${ }^{1}$ Peng Lei, ${ }^{1,5}$ Ming Liu ${ }^{1}$
}

To cite: Zhang S, Shu Y, Li W, et al. High haemoglobin levels and mortality in males with intracerebral haemorrhage: a retrospective cohort study. BMJ Open 2022;12:e048108. doi:10.1136/ bmjopen-2020-048108

- Prepublication history and additional supplemental material for this paper are available online. To view these files, please visit the journal online (http://dx.doi.org/10.1136/ bmjopen-2020-048108)

SZ, YS and WL contributed equally.

Received 29 December 2020 Accepted 24 November 2021

Check for updates

C) Author(s) (or their employer(s)) 2022. Re-use permitted under CC BY-NC. No commercial re-use. See rights and permissions. Published by BMJ.

For numbered affiliations see end of article.

Correspondence to

Professor Ming Liu; wyplmh@hotmail.com and

Dr Peng Lei;

peng.lei@scu.edu.cn

\section{ABSTRACT}

Objectives To examine the association between high haemoglobin levels and outcomes in intracerebral haemorrhage $(\mathrm{ICH})$ in a multicentre cohort study. Design Prospective multicentre cohort study. Settings 21 tertiary hospitals across mainland China. Participants A total of 5318 consecutive in-hospital spontaneous ICH patients were recruited between January 2012 and June 2016.

\section{Primary and secondary outcome}

measures Haemoglobin levels were measured on admission. Binary or ordinary logistic regression was used to evaluate the independent relationship of haemoglobin level with clinical outcomes at 3 months, measured as death or disability. Restricted cubic spline regression was fitted to examine the potential non-linear shape of the dose-response curve between the whole haemoglobin levels and 3-month poor outcomes.

Results A total of 5031 patients with ICH were analysed (64.3\% male; mean age (SD), 57.8 (15.2) years). We found that the highest haemoglobin quintile was associated with poor outcomes 3 months in males (adjusted OR (aOR) $1.65,95 \% \mathrm{Cl} 1.21$ to 2.25 ) but not in females, which was also observed in the pooled analysis of three subcohorts in male patients (average a0R $1.70,95 \% \mathrm{Cl} 1.23$ to 2.33). The spline regression suggested a non-linear association between haemoglobin levels and outcomes and a linear relationship was observed between an elevated haemoglobin level and 3-month disability/death in males (haemoglobin level per $10 \mathrm{~g} / \mathrm{L}$ : aOR $1.24,95 \% \mathrm{Cl} 1.10$ to $1.40, p<0.001$ ), which was mediated by larger haematoma volume (effect size: $0.115,95 \% \mathrm{Cl} 0.012$ to 0.231 ).

Conclusions This study found a sex-specific association between an elevated haemoglobin level and poor 3-month outcomes, which might be mediated by larger haematoma volume.

\section{INTRODUCTION}

Intracerebral haemorrhage (ICH) has the most unfavourable outcome among all types of stroke, and therefore represents a significant health burden. ${ }^{12}$ However, there is no specific treatment for ICH and biomarkers are of limited value to predict the outcome of the disorder. Reduced haemoglobin or anaemia is frequently reported to be associated with poor outcomes in patients with

\section{Strengths and limitations of this study}

- A multicentre cohort study included the large sample size and heterogeneous intracerebral haemorrhage patient population.

- Multivariable linear logistic analysis and sensitivity subgroup analysis based on meta-analyses pooled estimates were performed to draw the conclusion.

- Mediation analysis was performed to explore the mediator of the association between high haemoglobin levels and mortality.

- The haemoglobin levels based one measurement on admission might be prone to regression attenuation bias.

ICH. ${ }^{3-5}$ However, elevated haemoglobin levels are double-edged. Elevated haemoglobin accompanied with higher heme production, which was a prominent breakdown product of haemoglobin. In subarachnoid haemorrhage $(\mathrm{SAH})$, high levels of hemin were found in the cerebrospinal fluid during SAH-induced vasospasm and to activate an inducible isoform of nitric oxide synthase in vascular smooth-muscle cells. ${ }^{6}{ }^{7}$ Animal studies demonstrated that heme-induced oxidative stress and vascular expression of heme oxygenase-1, which impaired the platelet-dependent thrombosis process via stimulating production of Cyclic guanosine monophosphate (cGMP), an inhibitor of platelet aggregation. ${ }^{8-11}$ It was possible that the heme-induced platelet dysfunction might exacerbate poor outcomes in either in ischaemic stroke or in ICH.

In acute stroke, it was reported that an elevated haemoglobin concentration was associated with higher cardiovascular disease or stroke incidence. ${ }^{12-14}$ In ischaemic stroke, recent study also reported that the highest quartile of haemoglobin level or the elevated admission haemoglobin levels $(153 \mathrm{~g} / \mathrm{L}$ in male and $142 \mathrm{~g} / \mathrm{L}$ in female) was correlated with poorer 3-month or 1-year outcomes, 
respectively. ${ }^{14}{ }^{15}$ However, there was a lack of evidence concerning the relationship between the high haemoglobin levels and outcomes in ICH. Our aims were, therefore, to investigate in a large multicentre cohort of patients with ICH, the association between the elevated haemoglobin levels and outcomes and explore the possible underlying mechanisms.

\section{METHODS}

\section{Study design and participants}

We used a cohort study design based on a prospective, multicentre, hospital-based registry that collected data of patients with acute ICH admitted to 21 tertiary hospitals across a wide range of cities in China from January 2012 to June 2016. A total of 5318 patients who received a clinical diagnosis of spontaneous ICH confirmed by brain imaging were screened. The study was approved by the Biomedical Research Ethics Committee and the Committee on Human Research of West China Hospital, Sichuan University (2013 [124]). ${ }^{16}$

Patients with first-ever ICH were consecutively recruited. Patients were included if they (1) were at least 18 years old, (2) had been diagnosed with ICH based on non-contrast computed tomography (NCCT) performed within 72 hours from the presumed symptom onset and (3) had undergone CT or MRI to distinguish haemorrhagic stroke from ischaemic stroke. Patients underwent assessments at baseline (on admission) and at 3 months after stroke. Patients were excluded (1) if they were diagnosed with traumatic ICH, primary subdural/epidural haematoma, intracranial venous thrombosis or haemorrhage due to a tumour or recurrent ICH; (2) if they had stroke due to primary SAH with or without ICH and haemorrhagic transformation of a cerebral infarction or (3) haemoglobin measurements on admission were missing.

\section{Procedures}

Information about baseline demographic characteristics was obtained predominantly through in-person interviews. In-hospital details, including clinical features and diagnosis, were obtained through medical records and interviews with patients or their families. Follow-up details were obtained primarily through telephone interviews at 3 months after stroke. The state of consciousness was assessed by the Glasgow Coma Scale (GCS) Score with a score from 3 to 15 , with a lower score indicating a worse level of consciousness, and the severity of neurological deficits was assessed using National Institutes of Health Stroke Scale (NIHSS) Score from 0 to 42 , with higher scores indicating a more severe neurological deficit. CT scans of the head were performed in all patients on admission. The NCCT scans were acquired with a 5 mm-thick axial slice, 120-140 kVs (peak), 10-500 mA, and reviewed for determination of haematoma location and volume. haematoma volume was determined by the formula of ellipsoids $(\mathrm{A} \times \mathrm{B} \times \mathrm{C} / 2) .{ }^{17}$ Haemoglobin levels were measured on admission using the hemiglobincyanide method. Drinking was confirmed if the patients were weekly drinkers, those who usually drank alcohol at least once per week during the past 12 months. Smoking was confirmed if the patients were regular smokers, who admitted to smoke at least one cigarette (or equivalent) per day. The primary outcome was disability/ death at 3 months, defined by scores of 3-6 on the Modified Ranking Scale (mRS) and the follow-up period was 3 months. Subgroup was determined according to the geographic distribution of these subcentres. West China Hospital was allocated to cohort 1; the subcentres within Sichuan province were allocated to cohort 2; the subcentres of other provinces in China were allocated to cohort 3.

The follow-up period was 3 months with a follow-up rate of $88 \%(4418 / 5035)$. The baseline characteristics of the missing cases were not significantly different from those included (data not shown).

\section{Statistical analysis}

Categorical variables were presented as counts (\%), and the continuous or discrete variables were presented as mean (SD) or median (IQR). Student's t test, the $\chi^{2}$ test, Analysis of Variance (ANOVA), Mann-Whitney U test, Fisher's exact test and Kruskal-Wallis test were used for univariate analysis among groups with relevant variables as appropriate. Associations of clinical characteristics with death/disability were analysed using logistic regression models. Data are reported as ORs and 95\% CIs. Where appropriate, adjusted ORs (aORs) were reported. Age, premorbid antithrombotics use, hyperlipidemia, diabetes mellitus, alcohol consumption, GCS, NIHSS, haematoma volume and locations, blood creatine levels, blood glucose, activated partial thromboplastin time (APTT), prothrombin time (PT), fibrinogen, blood pressure, chronic kidney disease, lung disease, antihypertension treatment, cerebral oedema treatment (dehydration treatment) and surgical interventions were the selected potential confounders, which were adjusted in all multivariable models. To investigate the association between an elevated haemoglobin level and outcomes, the haemoglobin concentration was categorised into quintiles with similar sample numbers in each quintile $(126,137,148$ and $156 \mathrm{~g} / \mathrm{L}$ for males and $115,123,130$ and $139 \mathrm{~g} / \mathrm{L}$ for females). The forest plot of the estimates of the three subcohorts was performed using the Mantel-Haenszel random-effect model of the meta-analysis (Revman V.5.0). Restricted cubic spline regression was fitted to examine the potential non-linear shape of the doseresponse curve between the whole haemoglobin levels and 3-month poor outcomes and to check whether the breakpoints identified were in accordance with results of the haemoglobin quartiles. A threshold of $\mathrm{p}<0.10$ was used to declare statistical significance for heterogeneity. Two-sided $p$ values are reported, with $p<0.05$ considered statistically significant in all tests unless another threshold was given. Statistical analyses were performed in R Core 
Team (2017) (R: A language and environment for statistical computing. R Foundation for Statistical Computing, Vienna, Austria (https://wwwR-projectorg/). The calculations and plottings were conducted using the algorithm packages in R including splines, MASS, dplyr and ggplot2 packages.

Mediation analysis was performed to estimate whether haematoma volume (as the mediator) was the mediator for any relationship between haemoglobin level (independent variable) and poor outcome (dependent variable) by analysing all three variables together with four steps: (1) path c: the total effect of the an elevated haemoglobin level (group factor) on the outcome; (2) path a: the group effect on haematoma volume; (3) path b: the correlation between haematoma volume and outcomes, after controlling for the group factor and (4) the $a \times b$ effect, which was referred to as the indirect effect and was indicative of whether the predictor-outcome relationship was significantly reduced after controlling for the mediator. If all four tests reached the level of significance, haematoma volume was considered to significantly mediate the group effect on the poor outcome. The $\mathrm{a} \times \mathrm{b}$ indirect effect was evaluated using the PROCESS macro implemented in SPSS (Hayes, 2018). A bootstrap method with 5000 repetitions was used to estimate the CIs for the indirect effects. An empirical 95\% CI that did not include zero indicated significance at the 0.05 level. ${ }^{18}{ }^{19}$ Analyses were performed using SPSS (IBM, Armonk, New York).

\section{RESULTS}

A total of 5318 patients with spontaneous ICH were screened and 5031 patients were included in the final analysis (figure 1). Of the 5031 patients, $3233(64.3 \%)$ patients were male, the mean age was 57.8 (15.2) years (table 1). The 3-month mortality rate was $19.2 \%(19.5 \%$ for males and $18.8 \%$ for females) and 3-month disability/ death rate was $48.8 \%$ (49.3\% for males and $48.0 \%$ for females).

\section{Association of an elevated haemoglobin with poor outcomes in male patients with ICH across three different subcohorts}

Table 2 depicts the association of haemoglobin levels with 3-month disability/death by quintiles of sex-specific baseline haemoglobin levels. Compared with the male patients with haemoglobin levels of 138-146g/L, after adjusting for potential confounders, the haemoglobin level within the highest quintile was associated with higher poor outcomes (aOR 1.65, 95\% CI 1.21 to $2.25, \mathrm{p}=0.002$ ). Those significant associations between high haemoglobin levels and poor outcomes were not observed in the female patients.

To further confirm the association between the highest haemoglobin quintile $(>156 \mathrm{~g} / \mathrm{L})$ and 3-month poor outcomes in males, patients were divided into three different subcohorts: cohort 1, cohort 2 and cohort 3 according to the geographical distribution of the included medical subcentres and stratified by the
5318 Patients with acute spontaneous ICH diagnosis, age $\geq 18$, and enrolled from 21 tertiary hospitals

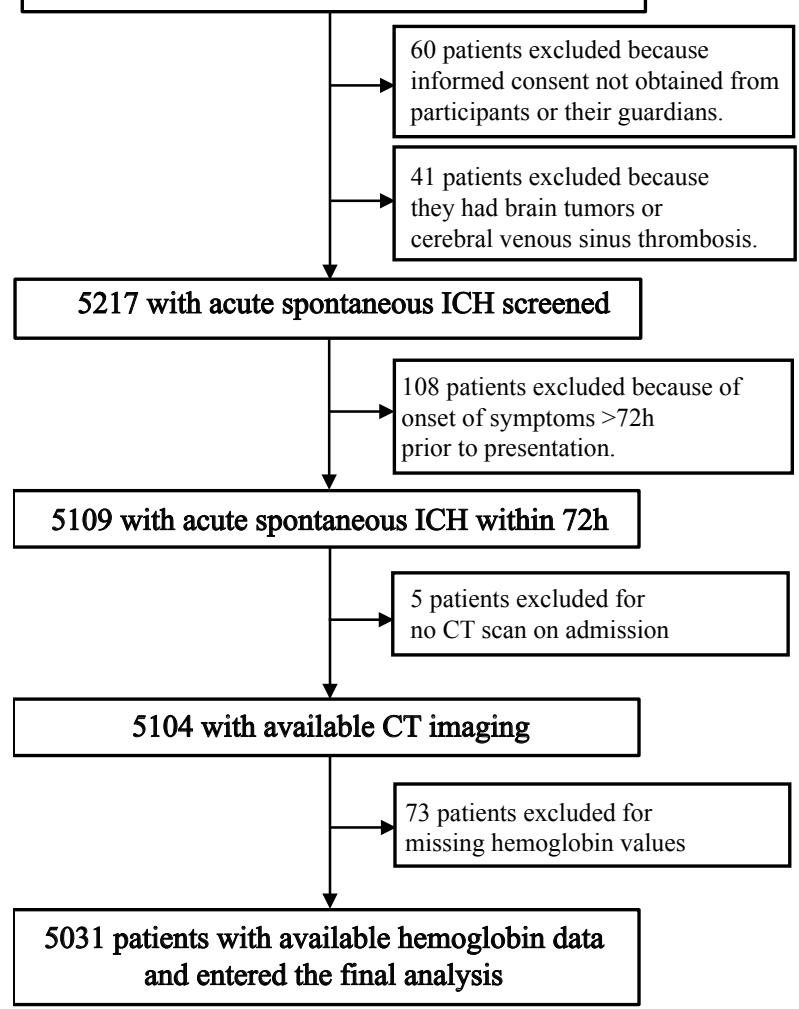

Figure 1 Flow chart of patients screened, describing both those included and excluded from the study. $\mathrm{ICH}$, intracerebral haemorrhage.

haemoglobin quintiles with the third quintile as the reference (figure 2). A significant association between the highest haemoglobin quintile and poor outcomes was observed in cohort 1 (aOR 2.08, 95\% CI 1.01 to 4.28) and cohort 3 (aOR 1.67, 95\% CI 1.10 to 2.55). In cohort 2 , there was a similar trend but not significant. Pooling and analysing the results from three subcohorts using the generic inverse variance method of meta-analysis, the highest quintile, compared with the reference, was associated with worse outcomes (average aOR 1.70, 95\% CI 1.23 to 2.33). In females, the association was not observed in any subcohorts (online supplemental figure 1).

Haemoglobin level linearly and negatively correlates with poor outcomes in male patients with an elevated haemoglobin level $(>156 \mathrm{~g} / \mathrm{L}$ ) via larger haematoma volume

The spline regression was employed to investigate any non-linear correlation between haemoglobin levels and outcomes. In the spline plot, the nadir of the curve was observed at a haemoglobin level of approximately $150 \mathrm{~g} / \mathrm{L}$ $\left(\mathrm{P}_{\text {non-linearity }}=0.004\right)$, as shown in figure $3 \mathrm{~A}$; however, the non-linear correlation was not found in female patients $\left(\mathrm{P}_{\text {non-linearity }}=0.818\right)$. The turning points of the U-shaped curve of male patients, beyond which there was a sharp increase in the prevalence of poor outcomes, were 
Table 1 Baseline characteristics of study population according to gender

\begin{tabular}{|c|c|c|c|}
\hline & Total & Female & Male \\
\hline Characteristics & $\mathrm{n}=5031$ & $n=1798$ & $n=3233$ \\
\hline Age, years & $57.8 \pm 15.2$ & $58.0 \pm 15.5$ & $57.6 \pm 15.1$ \\
\hline \multicolumn{4}{|c|}{ Comorbidities and risk factors, $\mathrm{n}(\%)$} \\
\hline HD history & $359(7.1 \%)$ & $142(7.9 \%)$ & $217(6.7 \%)$ \\
\hline Hypertension & 2965 (58.9\%) & $1081(60.1 \%)$ & $1884(58.3 \%)$ \\
\hline Hyperlipidemia & $1413(33.0 \%)$ & $521(34.2 \%)$ & $892(32.3 \%)$ \\
\hline Diabetes mellitus & $430(8.5 \%)$ & $151(8.4 \%)$ & $279(8.6 \%)$ \\
\hline Chronic kidney disease & $256(5.1 \%)$ & $58(3.2 \%)$ & $198(6.1 \%)$ \\
\hline Antithrombotics & $432(8.6 \%)$ & $130(7.2 \%)$ & $302(9.3 \%)$ \\
\hline Alcohol & $1068(21.2 \%)$ & $56(3.1 \%)$ & $1012(31.3 \%)$ \\
\hline Smoking & $1290(25.6 \%)$ & $70(3.9 \%)$ & $1220(37.7 \%)$ \\
\hline \multicolumn{4}{|l|}{ Clinical status } \\
\hline GCS & $13.0(8.0,15.0)$ & $13.0(8.0,15.0)$ & $13.0(8.2,15.0)$ \\
\hline NIHSS & $8.0(3.0,16.0)$ & $9.0(3.0,17.0)$ & $8.0(3.0,16.0)$ \\
\hline Haematoma volume, $\mathrm{mL}$ & $13.0(5.2,27.8)$ & $11.0(5.0,23.6)$ & $14.4(5.4,30.0)$ \\
\hline $\mathrm{SBP}, \mathrm{mm} \mathrm{Hg}$ & $162.3 \pm 31.3$ & $160.2 \pm 32.3$ & $163.4 \pm 30.7$ \\
\hline DBP, $\mathrm{mm} \mathrm{Hg}$ & $95.0 \pm 18.1$ & $92.7 \pm 17.8$ & $96.3 \pm 18.2$ \\
\hline Haemoglobin, g/L & $136 \pm 20$ & $127 \pm 16$ & $141 \pm 20$ \\
\hline HCT & $0.41 \pm 0.06$ & $0.38 \pm 0.05$ & $0.42 \pm 0.06$ \\
\hline Albumin, $g / L$ & $41.7(38.5,44.9)$ & $41.9(38.7,44.9)$ & $41.7(38.3,44.8)$ \\
\hline Platelet count, $10^{9} / \mathrm{L}$ & $161(121,207)$ & $168(123,216)$ & $158(119,202)$ \\
\hline $\mathrm{PT}, \mathrm{s}$ & $11.8(11.0,12.9)$ & $11.8(10.9,12.8)$ & $11.8(11.0,12.9)$ \\
\hline APTT, s & $26.5(23.6,30.0)$ & $26.1(23.2,29.5)$ & $26.7(23.8,30.3)$ \\
\hline Fibrinogen, $\mathrm{g} / \mathrm{L}$ & $2.8(2.3,3.5)$ & $2.9(2.4,3.5)$ & $2.8(2.3,3.5)$ \\
\hline INR & $1.02(0.95,1.10)$ & $1.01(0.94,1.09)$ & $1.02(0.95,1.10)$ \\
\hline Blood glucose, $\mathrm{mmol} / \mathrm{L}$ & $7.25(6.03,9.12)$ & $7.34(6.14,9.40)$ & $7.20(6.00,9.01)$ \\
\hline Creatine, $\mu \mathrm{mol} / \mathrm{L}$ & $70.0(57.0,85.8)$ & $58.0(48.9,71.0)$ & $76.0(64.9,91.0)$ \\
\hline \multicolumn{4}{|l|}{ Haematoma location, n (\%) } \\
\hline BG or thalamus & $2969(64.1 \%)$ & 1053 (63.9\%) & $1916(64.2 \%)$ \\
\hline Lobar & $1626(33.7 \%)$ & $575(33.3 \%)$ & $1051(33.8 \%)$ \\
\hline Brainstem & $405(8.4 \%)$ & $135(7.8 \%)$ & $270(8.7 \%)$ \\
\hline Cerebellar & $292(6.0 \%)$ & $101(5.8 \%)$ & $191(6.2 \%)$ \\
\hline IVH & $1632(33.8 \%)$ & $601(34.8 \%)$ & $1031(33.2 \%)$ \\
\hline \multicolumn{4}{|c|}{ Therapies and complications, $\mathrm{n}(\%)$} \\
\hline Antihypertension & 2826 (58.9\%) & $978(57.8 \%)$ & $1848(59.5 \%)$ \\
\hline Cerebral oedema treatment & $4285(89.3 \%)$ & $1516(89.7 \%)$ & $2769(89.2 \%)$ \\
\hline Surgical intervention & $1354(26.9 \%)$ & $500(27.8 \%)$ & $854(26.4 \%)$ \\
\hline Respiratory infection & $1097(21.8 \%)$ & $344(19.1 \%)$ & $753(23.3 \%)$ \\
\hline
\end{tabular}

Descriptive statistics were calculated using mean \pm SD or median (IQR) for continuous variables and frequencies for categorical variables. APTT, activated partial thromboplastin time; BG, basal ganglia; DBP, diastolic blood pressure; GCS, Glasgow Coma Scale; HCT, haematocrit; $\mathrm{HD}$, heart disease; INR, international normalised ratio; IVH, intraventricular haemorrhage; NIHSS, National Institutes of Health Stroke Scale; PT, prothrombin time; SBP, systolic blood pressure.

approximately between 150 and $160 \mathrm{~g} / \mathrm{L}$, which was in accordance with the results of the haemoglobin quartiles.

We compared the potential risk factors of male patients with different haemoglobin quintiles. Compared with reference quintile, the patients with the highest haemoglobin quintile were characterised with younger age (51.0 years), higher frequency of diabetes $(62.2 \%)$, higher alcohol consumption (36.7\%), higher platelet count 
Table 2 Quintiles of haemoglobin on admission and 3-month disability/death in male and female patients with ICH

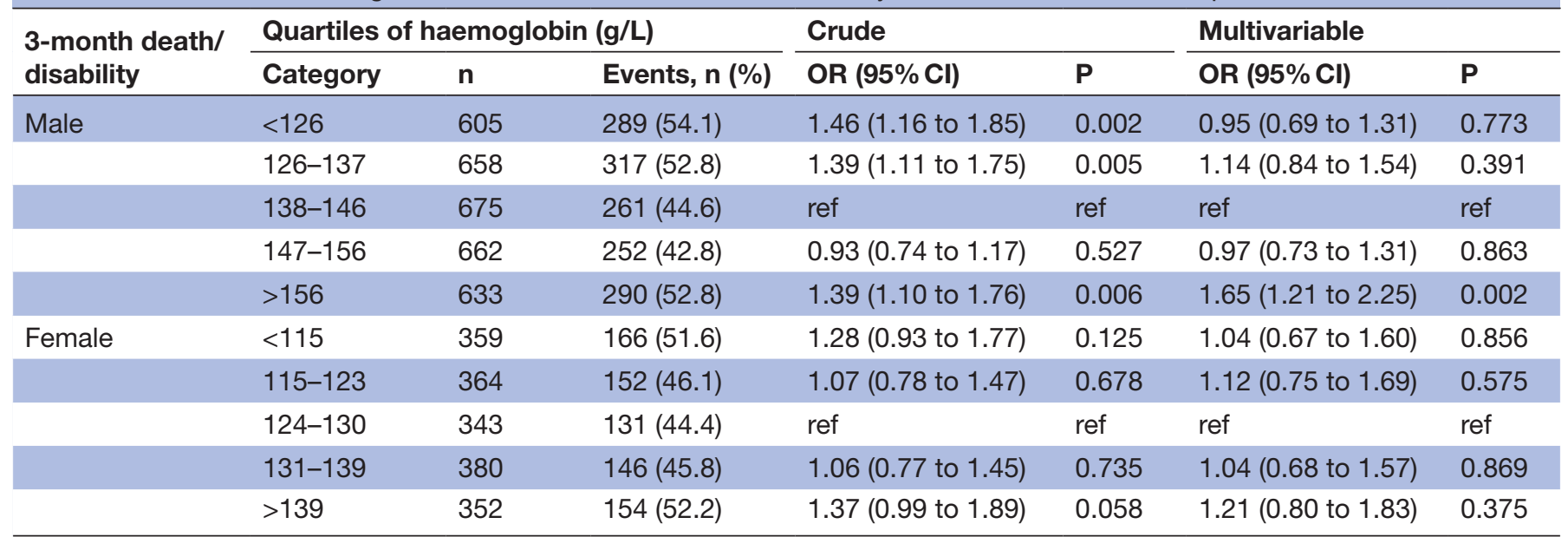

All models were adjusted for age, premorbid antithrombotics use, hyperlipidemia, diabetes mellitus, alcohol consumption, GCS, NIHSS, haematoma volume and locations, blood creatine levels, blood glucose, activated partial thromboplastin time, prothrombin time, fibrinogen, blood pressure, chronic kidney disease, lung disease, antihypertension treatment, cerebral oedema treatment and surgical interventions. GCS, Glasgow Coma Scale; ICH, intracerebral haemorrhage; NIHSS, National Institutes of Health Stroke Scale.

$\left(171 \times 10^{9} / \mathrm{L}\right)$, higher haematoma volume $(15.4 \mathrm{~mL})$, higher frequency of antihypertensive $(66.4 \%)$ and cerebral oedema treatment $(92.4 \%$; online supplemental table 1), which were all adjusted in the following analysis as potential confounders. When we analysed the linear correlation between haemoglobin levels and outcomes in the patients with haemoglobin levels of the highest quartile $(>156 \mathrm{~g} / \mathrm{dL})$, a linear relationship was observed between an elevated haemoglobin level and 3-month disability/death in males (haemoglobin level per $10 \mathrm{~g}$ / $\mathrm{dL}$ : aOR $1.24,95 \%$ CI 1.10 to $1.40, \mathrm{p}<0.001$ ), but not in females $(\mathrm{p}=0.700$; figure $3 \mathrm{~B})$.

To investigate the potential mediators between an elevated haemoglobin level and poor outcome, we performed a mediation analysis in male ICH patients with haemoglobin level $>156 \mathrm{~g} / \mathrm{L}$ (figure 4). We define path $\mathrm{a}$, path $\mathrm{b}$ and path $\mathrm{c}$ as group difference in haematoma volume, group-independent haematoma-outcomes relations and group difference in 3-month disability/death, respectively. Given the requirement for a significant path $c$, path a and path $\mathrm{b}$, there was only one candidate mediation path, in which the group factor, haematoma volume and 3-month disability/death are the predictor, mediator and outcome, respectively (path c', $\beta=0.398, p=0.005$; path a, $\beta=3.20, p=0.031$; path $b, \beta=0.036$, $p<0.001)$. The bootstrap simulation $(\mathrm{n}=5000)$ confirmed a significant indirect effect (effect size: $a \times b=0.115,95 \%$ CI 0.012 to $0.231, \mathrm{p}<0.05)$, implying that 3-month death was expected to increase by 0.115 for every $10 \mathrm{~g} / \mathrm{L}$ increase in haemoglobin when haemoglobin levels were more than $156 \mathrm{~g} / \mathrm{L}$, if one considers only the indirect influence via haematoma volume. Therefore, an elevated haemoglobin level might impair 3-month disability/death through larger haematoma volume.

\section{DISCUSSION}

In a prospective cohort of 5031 consecutive intracerebral haemorrhage patients, we found that the association of the highest haemoglobin level with an increased 3-month poor outcome risk was observed in males but not in females. There was a linear association among men such that every $10 \mathrm{~g} / \mathrm{L}$ increment of haemoglobin level was associated with 1.24-fold of increased 3-month poor outcomes risk when haemoglobin was more than $156 \mathrm{~g} / \mathrm{L}$.

Elevated haemoglobin levels have been reported to be positively associated with a higher risk of various cardiovascular diseases. A study retrospectively analysed a cohort including 170078 men and 122116 women without cardiovascular diseases, which showed that, in men, an increased haemoglobin concentration outside $\geq 160 \mathrm{~g} / \mathrm{L}$ was associated with an increase in all-cause mortality risk (adjusted HR:1.10, 95\% CI 1.01 to 1.20 ), the trend was similar in women but less significant. ${ }^{13}$ In ischaemic stroke, Guo et al reported that in a cohort of 3881 patients, the highest quartile of haemoglobin level was associated with an increased 3-month major disability and death rate (adjusted ORs 1.49, 95\% CI 1.11 to 1.99). ${ }^{14}$ Tanne et al investigated haemoglobin concentration and 1-year outcome among 859 consecutive patients with acute stroke and found that elevated admission haemoglobin level was correlated with a higher risk of death in stroke $(153 \mathrm{~g} / \mathrm{L}$ in male and $142 \mathrm{~g} / \mathrm{L}$ in female $){ }^{15}$ however, only $15.4 \%$ patients of the cohort had an ICH. In contrast, Park et al reported that in a cohort of 2681 consecutive patients with acute ischaemic stroke, poor outcome was not related to the higher end of the haemoglobin range. ${ }^{20}$ In summary, reports regarding the relationship between higher haemoglobin levels and outcomes of cerebrovascular disease are inconsistent and most ICH studies were limited by small sample size. Our results support that, in 
A.

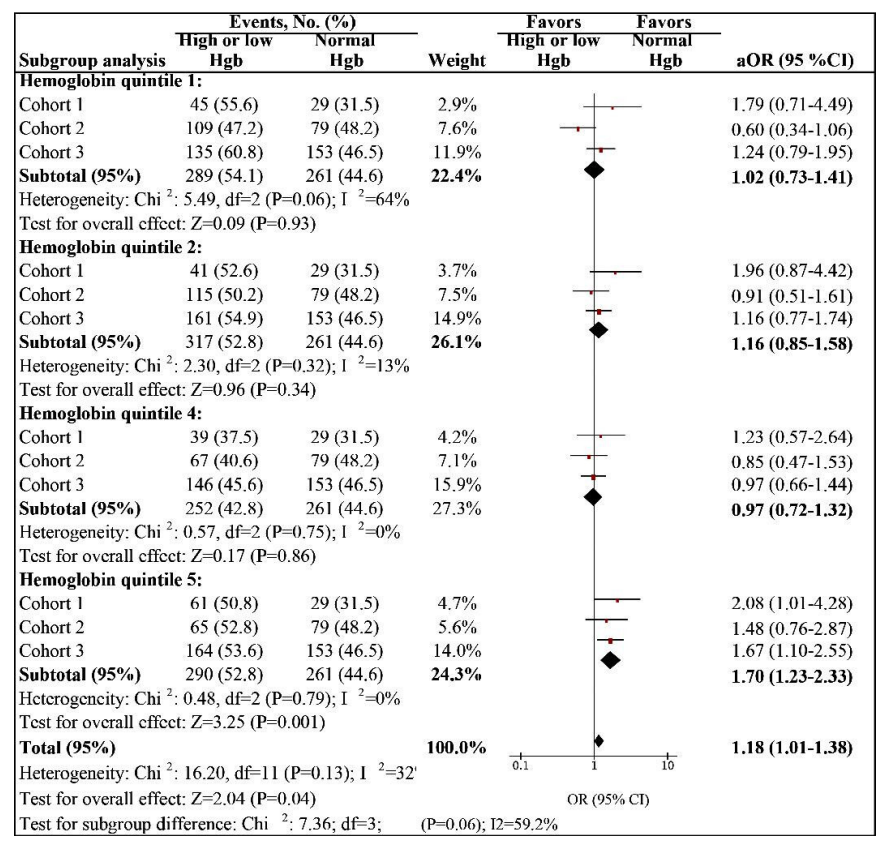

Figure 2 Pooled analysis of subcohorts in male patients with intracerebral haemorrhage. The haemoglobin concentration was categorised into quintiles by 126, 137, 148 and $156 \mathrm{~g} / \mathrm{L}$ in males. Forest plots of adjusted ORs for male $\mathrm{ICH}$ patients with other haemoglobin quintiles, compared with the third haemoglobin quintile, by subcohorts for 3-month disability/death. All models were adjusted for age, premorbid antithrombotics use, hyperlipidemia, diabetes mellitus, alcohol consumption, GCS, NIHSS, haematoma volume and locations, blood creatine levels, blood glucose, activated partial thromboplastin time, prothrombin time, fibrinogen, blood pressure, chronic kidney disease, lung disease, antihypertension treatment, cerebral oedema treatment and surgical interventions. aOR, adjusted OR; GCS, Glasgow Coma Scale; Hgb, haemoglobin level; ICH, intracerebral haemorrhage; NIHSS, National Institutes of Health Stroke Scale.

male patients with ICH, the highest quintile of haemoglobin level was associated with poor outcomes. Further prospective studies from other samples of patients with ICH are needed to validate our findings.

We also found that haematoma volume mediated the association between an elevated haemoglobin level and poor outcomes in ICH. The pathophysiologic mechanism for this might be the result of multiple factors, such as the hemin-induced platelet-dependent thrombosis dysfunction or the iron toxicity and its related inflammation and coagulopathy. ${ }^{821-23}$ Although iron overload is not directly associated with high haemoglobin levels, however, iron dysregulation could be found in haematological disease such as polycythemia vera. A possible explanation of the larger haematoma volume could be similar to that found in the less frequent haemorrhagic presentations of patients with polycythemia vera or other iron-induced abnormal blood coagulation. ${ }^{24}$ We noted that, although the platelet was higher in these patients, the coagulation function measured by PT, APTT and fibrinogen was not

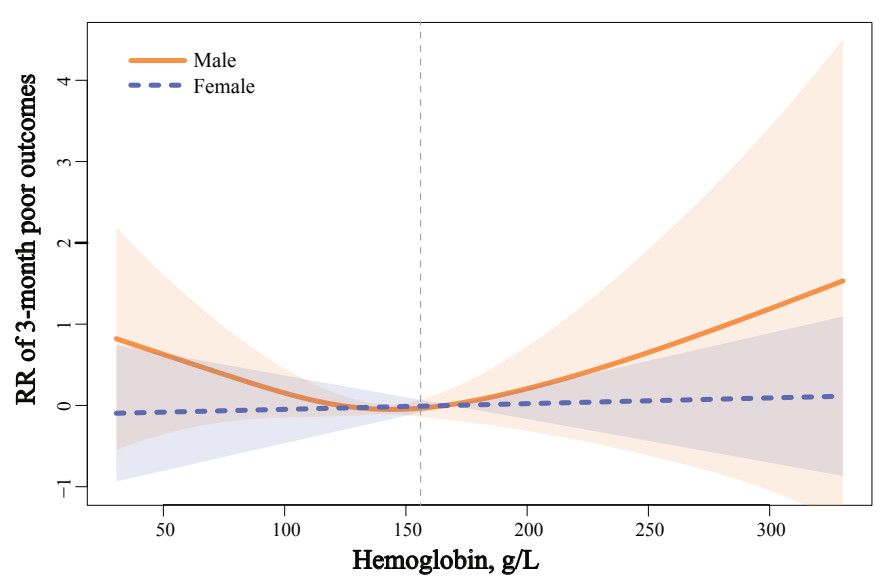

B.

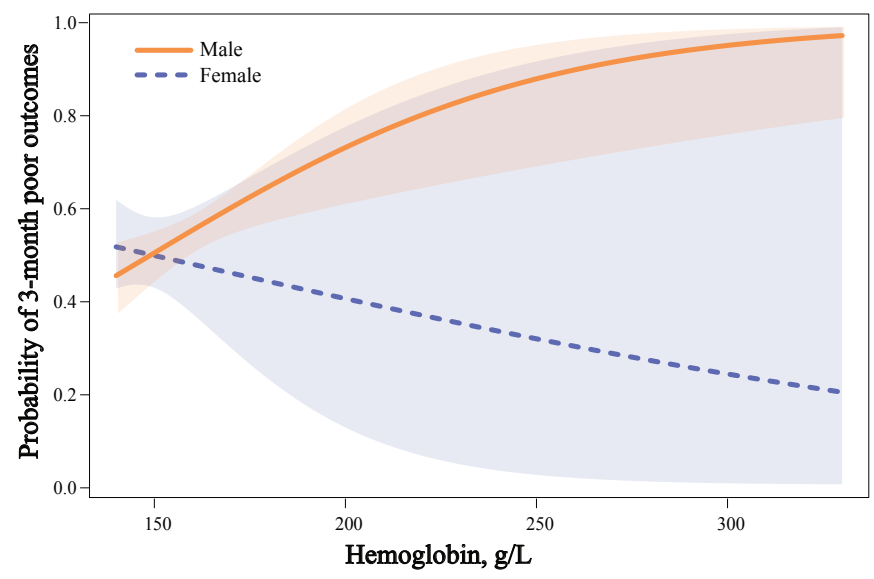

Figure 3 A. The nonlinear correlation was found in male patients with a nadir value between 150 and $160 \mathrm{~g} / \mathrm{L}$; B. Predicted poor 3-month outcome probabilities by baseline haemoglobin levels in male and female ICH patients with an elevated haemoglobin level $(>156 \mathrm{~g} / \mathrm{L})$. All models were adjusted for age, premorbid antithrombotics use, hyperlipidemia, diabetes mellitus, alcohol consumption, GCS, NIHSS, haematoma volume and locations, blood creatine levels, blood glucose, activated partial thromboplastin time, prothrombin time, fibrinogen, blood pressure, chronic kidney disease, lung disease, antihypertension treatment, cerebral oedema treatment and surgical interventions. The grey dashed line indicated a haemoglobin level at 156/dL. GCS, Glasgow Coma Scale; ICH, intracerebral haemorrhage; NIHSS, National Institutes of Health Stroke Scale.

significantly different from the reference group, indicating that other factors or coagulation pathways might be underlying the observation. In the future, it would be interesting to study if there would be some haematological aspects associated with high haemoglobin levels.

Our findings also suggested a gender difference in the association between elevated haemoglobin levels and poor outcomes. The gender differences we observed could be partly explained by the different haemoglobin levels between men and women. We observed a wider haemoglobin distribution in males with a median value of $140 \mathrm{~g} / \mathrm{L}$, compared with a more focused distribution 

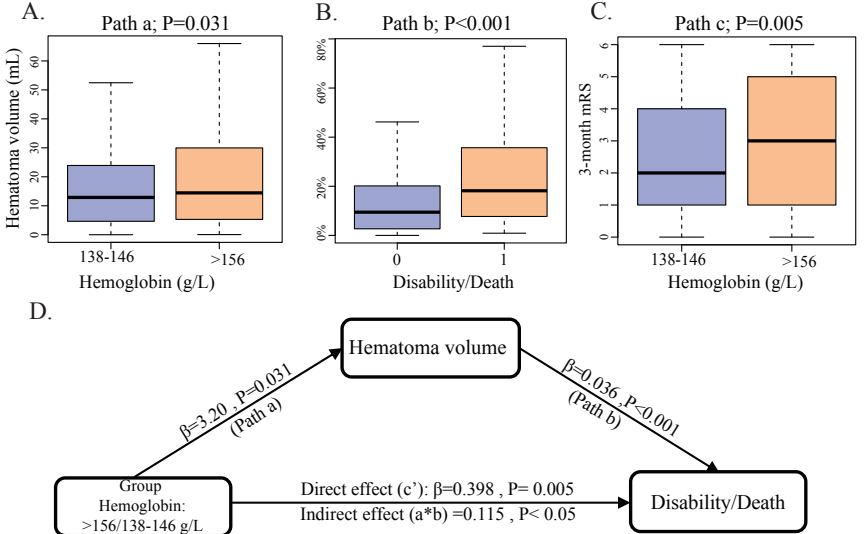

Figure 4 Mediation model of an elevated haemoglobinhaematoma volume-3-month disability/death pathway in male $\mathrm{ICH}$ patients. (A) Path a: Significant elevated haemoglobin $(>156 \mathrm{~g} / \mathrm{L})$ group effect on haematoma volume. (B) Path b: Significant haematoma volume-disability/death correlation after controlling for group factor, age and urea nitrogen. (C) Path c: Significant elevated haemoglobin (>156 g/L) group effect on 3-month mRS (disability/death). (D) Schematic diagram of the mediation model of the pathway from an elevated haemoglobin (>156 g/L) to larger haematoma volume to disability/death in the male patients with $\mathrm{ICH}$. ICH, intracerebral haemorrhage; mRS, Modified Ranking Scale.

in females with a median value of $128 \mathrm{~g} / \mathrm{L}$ (online supplemental figure 2). It is plausible that the dispersed distribution of haemoglobin in males might partly explain why male ICH patients were more vulnerable to elevated haemoglobin than females. In females, repeatedly altered haemoglobin status caused by menstruation might make the women's cardiovascular system resistant to the change of haemoglobin levels. ${ }^{25}$ Similar to our results, the Apolipoprotein Mortality Risk Study reported that elevated haemoglobin levels were associated with acute myocardial infarction only in men. ${ }^{26}$ Another study analysing 18413 participants over a mean $7 \pm 2$ years of follow-up demonstrated that the highest quartiles of haemoglobin in both gender $(154 \mathrm{~g} / \mathrm{L}$ in male and $140 \mathrm{~g} / \mathrm{L}$ in female) were associated with higher risk of incident stroke (HR, 1.59, $95 \%$ CI 1.08 to 2.35$).{ }^{27}$ So far, the sex differences on the effect of elevated haemoglobin levels on various cardiovascular diseases has been still inconsistent. Further studies with large sample or individual patient data metaanalysis concerning the issue are needed.

Based on our multivariable analysis, an elevated haemoglobin level in male $(>156 \mathrm{~g} / \mathrm{L})$ could be an independent risk factor in male ICH patients. Therefore, haemodilution therapy aiming to decrease the haemoglobin levels might be beneficial to improve the prognosis in those patients. Moreover, mannitol-induced osmotic diuresis may cause or worsen dehydration, hypovolaemia and haemoconcentration. A rodent model study suggested that using mannitol during active ICH may interfere with collagen-stimulated platelet aggregation. ${ }^{28}$ Considering the routine use of mannitol in ICH treatment, caution should be exercised when using mannitol in men with an elevated haemoglobin concentration. Further studies on whether mannitol should be cautiously applied in ICH patients with elevated haemoglobin levels are needed.

Recently, Acosta et al did an exploratory analysis of two randomised clinical trials and one multiethnic observational study and found that higher haemoglobin levels were associated with better outcome in $\mathrm{ICH}^{29}$ The study did not perform the analysis based on male and female subgroup. Moreover, the different conclusion might be the result of the different ethnic groups, different distribution of outcomes as well as the strict exclusion criteria of both randomised clinical trials. Future individual patient data meta-analysis is needed to evaluate the high haemoglobin levels as the predictor of outcome.

Strengths of our study include the large and heterogeneous patient population which had rigorous prospective and systemic evaluations early after the onset of acute ICH. Nevertheless, some limitations need to be mentioned. First, we only included tertiary care hospitals in our study, which might lead to missing patients with less severe ICH. Second, the haemoglobin level was evaluated based on one single measurement on admission with various intervals from symptom onset to hospitalisation and thus prone to regression attenuation bias because this was based only on one measurement at presentation.

\section{CONCLUSION}

In summary, in this multicentre cohort of ICH, we revealed a sex-specific association between elevated haemoglobin levels and a poor 3-month outcome. Our data also suggested that haematoma volume might mediate the linear association between the haemoglobin levels and worse outcomes in male ICH patients with a haemoglobin level $>156 \mathrm{~g} / \mathrm{L}$.

\section{Author affiliations}

${ }^{1}$ Department of Neurology, Sichuan University West China Hospital, Chengdu, China ${ }^{2}$ State Key Laboratory of Biotherapy, West China Hospital, Sichuan University, Chengdu, China

${ }^{3}$ Department of Neurology, West China College of Nursing, Sichuan University West China Hospital, Chengdu, Sichuan, China

${ }^{4}$ West China College of Nursing, Sichuan University West China Hospital, Chengdu, Sichuan, China

${ }^{5}$ Department of Neurology, Sichuan University, Chengdu, China

Correction notice This article has been corrected since it first published. Peng Lei has been added as the corresponding author.

Contributors $M L$ is the guarantor of the article. $M L$ design and conduct the cohort and wrote the paper. ML, SZ and PL design and conduct the cohort study; SZ, YS, $\mathrm{YC}, \mathrm{CW}$ and $\mathrm{AD}$ collect the data and construct the database and $\mathrm{SZ}, \mathrm{YS}$ and $\mathrm{WL}$ analyze the data and write the paper.

Funding This study was funded by the National Key Research and Development Program of China of the Ministry of Science and Technology of China (2016YFC1300500-505 and 2018YFC1312300-303), by the National Natural Science Foundation of China (81870859, 92049115 and 8150092$)$ and by the 1.3 .5 project for disciplines of excellence, West China Hospital, Sichuan University (ZYG D18009).

Competing interests None declared.

Patient consent for publication Consent obtained from parent(s)/guardian(s). 
Ethics approval This study involves human participants and was approved by the Biomedical Research Ethics Committee and the Committee on Human Research of West China Hospital, Sichuan University (2013 (124)). Participants gave informed consent to participate in the study before taking part.

Provenance and peer review Not commissioned; externally peer reviewed.

Data availability statement The data that support the findings of this study are available from the corresponding author upon reasonable request.

Supplemental material This content has been supplied by the author(s). It has not been vetted by BMJ Publishing Group Limited (BMJ) and may not have been peer-reviewed. Any opinions or recommendations discussed are solely those of the author(s) and are not endorsed by BMJ. BMJ disclaims all liability and responsibility arising from any reliance placed on the content. Where the content includes any translated material, BMJ does not warrant the accuracy and reliability of the translations (including but not limited to local regulations, clinical guidelines, terminology, drug names and drug dosages), and is not responsible for any error and/or omissions arising from translation and adaptation or otherwise.

Open access This is an open access article distributed in accordance with the Creative Commons Attribution Non Commercial (CC BY-NC 4.0) license, which permits others to distribute, remix, adapt, build upon this work non-commercially, and license their derivative works on different terms, provided the original work is properly cited, appropriate credit is given, any changes made indicated, and the use is non-commercial. See: http://creativecommons.org/licenses/by-nc/4.0/.

\section{ORCID iDs}

Shuting Zhang http://orcid.org/0000-0001-6932-0131

Chenchen Wei http://orcid.org/0000-0002-8181-9696

\section{REFERENCES}

1 Hemphill JC, Greenberg SM, Anderson CS, et al. Guidelines for the management of spontaneous intracerebral hemorrhage: a guideline for healthcare professionals from the American heart Association/ American stroke association. Stroke 2015;46:2032-60.

2 van Asch CJ, Luitse MJ, Rinkel GJ, et al. Incidence, case fatality, and functional outcome of intracerebral haemorrhage over time, according to age, sex, and ethnic origin: a systematic review and meta-analysis. Lancet Neurol 2010;9:167-76.

3 Kuramatsu JB, Gerner ST, Lücking $\mathrm{H}$, et al. Anemia is an independent prognostic factor in intracerebral hemorrhage: an observational cohort study. Crit Care 2013;17:R148.

4 Kumar MA, Rost NS, Snider RW, et al. Anemia and hematoma volume in acute intracerebral hemorrhage. Crit Care Med 2009;37:1442-7.

5 Diedler J, Sykora M, Hahn P, et al. Low hemoglobin is associated with poor functional outcome after non-traumatic, supratentorial intracerebral hemorrhage. Crit Care 2010;14:R63.

6 Suzuki S, Kassell NF, Lee KS. Hemin activation of an inducible isoform of nitric oxide synthase in vascular smooth-muscle cells. $J$ Neurosurg 1995;83:862-6.

7 Suzuki M, Ogawa A, Asahara $\mathrm{H}$, et al. Nitric oxide and vasospasm. J Neurosurg 1997;86:741-2.

8 Peng L, Mundada L, Stomel JM, et al. Induction of heme oxygenase-1 expression inhibits platelet-dependent thrombosis. Antioxid Redox Signal 2004;6:729-35.

9 Praticó D, Pasin M, Barry OP, et al. Iron-Dependent human platelet activation and hydroxyl radical formation: involvement of protein kinase C. Circulation 1999;99:3118-24.
10 Gáll T, Balla G, Balla J, Heme BJ. Heme, heme oxygenase, and endoplasmic reticulum Stress-A new insight into the pathophysiology of vascular diseases. Int J Mol Sci 2019;20. doi:10.3390/ ijms20153675. [Epub ahead of print: 26 Jul 2019].

11 Fredenburgh LE, Merz AA, Cheng S. Haeme oxygenase signalling pathway: implications for cardiovascular disease. Eur Heart $J$ 2015;36:1512-8.

12 Zakai NA, Katz R, Hirsch C, et al. A prospective study of anemia status, hemoglobin concentration, and mortality in an elderly cohort: the cardiovascular health study. Arch Intern Med 2005;165:2214-20.

13 Lee G, Choi S, Kim K, et al. Association of hemoglobin concentration and its change with cardiovascular and all-cause mortality. J Am Heart Assoc 2018;7. doi:10.1161/JAHA.117.007723. [Epub ahead of print: 2901 2018].

14 Guo D, Zhu Z, Zhong C, et al. Hemoglobin level and three-month clinical outcomes among ischemic stroke patients with elevated systolic blood pressure. J Neurol Sci 2019;396:256-61.

15 Tanne D, Molshatzki N, Merzeliak O, et al. Anemia status, hemoglobin concentration and outcome after acute stroke: a cohort study. BMC Neurol 2010;10:22

$16 \mathrm{~mJ} \mathrm{~L}$, xiaoqun; You chao. The importance of the etiological study and standard etiological diagnosis in intracerebral hemorrhage. Chinese Journal of Neurology 2013;6:361-4.

17 Kothari RU, Brott T, Broderick JP, et al. The ABCs of measuring intracerebral hemorrhage volumes. Stroke 1996;27:1304-5.

18 Preacher KJ, Hayes AF. SPSS and SAS procedures for estimating indirect effects in simple mediation models. Behav Res Methods Instrum Comput 2004;36:717-31.

19 Valeri L, Vanderweele TJ. Mediation analysis allowing for exposuremediator interactions and causal interpretation: theoretical assumptions and implementation with SAS and SPSS macros. Psychol Methods 2013;18:137-50.

20 Park YH, Kim BJ, Kim J-S, et al. Impact of both ends of the hemoglobin range on clinical outcomes in acute ischemic stroke. Stroke 2013;44:3220-2.

21 Popov VM, Vladareanu AM, Bumbea $\mathrm{H}$, et al. Hemorrhagic risk due to platelet dysfunction in myelodysplastic patients, correlations with anemia severity and iron overload. Blood Coagul Fibrinolysis 2015;26:743-9

22 Dahi AA, Hanafy E, Al Pakra M. Iron overload and platelet function defects: possible correlation. J Investig Med High Impact Case Rep 2016;4:2324709616675645.

23 Lok J, Leung W, Murphy S, et al. Intracranial hemorrhage: mechanisms of secondary brain injury. Acta Neurochir Suppl 2011;111:63-9.

24 Lipinski B, Pretorius E. Novel pathway of iron-induced blood coagulation: implications for diabetes mellitus and its complications. Pol Arch Med Wewn 2012;122:115-22.

25 Terplan M. Menstruation and cardiovascular vulnerability. Arch Intern Med 2005;165:120.

26 Holme I, Aastveit AH, Hammar N, et al. High blood hemoglobin concentration as risk factor of major atherosclerotic cardiovascular events in 114,159 healthy men and women in the apolipoprotein mortality risk study (AMORIS). Ann Med 2012;44:476-86.

27 Panwar B, Judd SE, Warnock DG, et al. Hemoglobin concentration and risk of incident stroke in community-living adults. Stroke 2016;47:2017-24.

28 Liu J, Gao B-B, Clermont AC, et al. Hyperglycemia-Induced cerebral hematoma expansion is mediated by plasma kallikrein. Nat Med 2011;17:206-10.

29 Acosta JN, Leasure AC, Kuohn LR, et al. Admission hemoglobin levels are associated with functional outcome in spontaneous intracerebral hemorrhage. Crit Care Med 2021;49:828-37. 